\title{
Differential Games with (A) symmetric Players and Heterogeneous Strategies
}

\author{
Benteng Zou*
}

CREA, University of Luxembourg, Luxembourg

\begin{abstract}
One family of heterogeneous strategies in differential games with (a)symmetric players is developed in which one player adopts an anticipating open-loop strategy and the other adopts a standard Markovian strategy. Via conjecturing principle, the anticipating open-loop strategic player plans her strategy based on the possible updating the rival player may take. These asymmetric strategies should be appropriate choices in some modelling circumstances and they frame one of the infinitely many non-degenerate Markovian Nash Equilibrium. Except the stationary path, this kind of strategy makes the study of short-run trajectory possible, which usually are not subgame perfect. However, the shortrun non-perfection may provide very important policy suggestions.
\end{abstract}

Keywords: Differential game, Heterogeneous strategy, subgame perfect Markovian Nash Equilibrium, anticipating open-loop strategy.

\section{INTRODUCTION}

There are many economic examples in which it makes sense to assume that players do not have equal levels of commitment, and modelling this via different requirements imposed on the optimality of strategies open-loop on one end, Markovian best-reply on the other - seems like the right way to go. Economists and game theorists notice this idea for some time, but the application of this idea is quite limited.

In their seminal paper, Reinganum and Stokey (1985, p.162) state that when formulating a model, care should be taken to choose a strategy space that is appropriate for the situation under study. "Path strategies may be appropriate in some situations, decision rule strategies in others, and intermediate formulations in still others". More recently, Dockner et al. (2000, p.87) emphasize again that the analysis should "...consider equilibria in which some of the players represent their optimal control paths in openloop form while others choose nondegenerate Markovian strategies. " and further "...the choice to solve a differential game... ( for equilibria in which some players use open-loop strategies while others employ nondegenerate Markovian strategies) is part of the modelling stage and one should try to analyze that equilibrium which describes best the situation at hand".

This paper takes special care of these intermediate formulations in differential games, where players taking different strategic spaces seems more appropriate for

*Address of correspondence to this author at the $162 \mathrm{a}$, Avenue de la Faencerie, L-1511 Luxembourg; E-mail: benteng.zou@uni.lu

JEL classification: $\mathrm{C} 73, \mathrm{C} 72$. some modelling circumstances. We name heterogeneous strategic Markivian Nash equilibrium as one equilibrium where one player employs open-loop strategy and the other player takes standard Markovian strategy.

Here, we assume that all players share the same information structure ${ }^{1}$. Nevertheless, heterogeneity may appear in different aspects; thus appropriate strategy spaces should be chosen carefully.

First, players share the same information set, however, they may have difficulties to perform the same strategies as their rivals. Han et al. (2014), see Section 3 for more detail, present one such kind of situation in dynamic tax/infrastructure competition between large and small economies. As they argued that the small economy may be less efficient than the large one in interpreting its policy and offering public services, while the small economy may be more flexible than its large rival in collective or single-minded action. Dawid and Feichtinger (1996) show another such kind of asymmetric case in the study of optimal allocation of drug control efforts. In their model, both government (the drug controller) and drug dealers know the drug users, and their strategy spaces may differ.

Second, though players share the same information and have the same capacities to interpret the same strategies or policies as their rivals, they may differ in their moral standard or their attitudes. In other words,

\footnotetext{
${ }^{1}$ See more detail about information structure in Section 6.2 of Bacard and Olsder (1998). We do not study the case of information non-uniqueness as Basar (1976) and more recently Sorger (2015).
} 
players can be symmetric in their characteristics but just play asymmetric strategies. This can be seen from the international $\mathrm{CO} 2$ emission control problem. Despite the well-known stragedy of the commons results, unilateral action has been observed in collective-action problems like climate-change negotiations. Notable in 2007, EU country representatives committed themselves to a unilateral 20 per cent reduction in GHG emissions by 2020 and even offered a 30 per cent decrease in case an international agreement could be found. While the other big players (like the USA and China) can deviate from their commitments (or do not commit at all) and regularly revise their targets and policies. Benchekroun (2003) works out a situation of "Unilateral production restrictions in a dynamic duopoly". Though the setting of Benchekroun (2003) could be solve by the strategy provided here, he did in a quite different way. Our results could demonstrate another group of asymmetric Markovian Nash equilibrium. Indeed, under a different setting, Reinganum (1981) shows that under her setting two identical players adopting asymmetric strategies is optimal.

Thus, player engaging in this kind of differential games must first figure out that, among other important issues, in which strategy space she is playing, the subgame perfection of the chosen strategies and the possibility of studying the trajectory dynamics. Via conjecturing principle ${ }^{2}$, we shall introduce heterogeneous strategies by allowing some players to adopt open-loop strategies while others nondegenerate Markovian strategies.

The main contribution of this work is that Hamiltonian can be applied to look for heterogeneous strategic Nash Equilibrium along the whole trajectory. The conjecturing principle makes this possible. Otherwise, without this guessing principle, applying Hamiltonian searching for feedback strategies, we will face the difficulties of guessing each others' infinitely many optimal strategies, as clearly stated by Kamien and Schwartz (2003, P.275): " finding player i's optimal feedback strategy $u_{i}^{*}(x, t)$ requires that player j's optimal feedback strategy $u_{j}^{*}(x, t)$ be known which, in turn, requires that player i's optimal be known, and so on." The family of games, where one player is less flexible in changing strategies based on state of the world than the other player, makes this guessing process stop at one step instead of infinitely many.

${ }^{2}$ Guessing technique is often in use in game theory and especially differential games, see Long (2010) for more examples.
Our approach fits specially well to differential games with unilateral commitment. Most of the economic literature $^{3}$ applying differential games ignores the impact of unilateral commitment and focuses only on symmetrical strategy spaces. Nevertheless, unilateral commitment is not the only case where these kinds of heterogeneous strategies are adopted. In Section 3, we present different situations where this kind of strategy space should be taken. Thus far, the literature provides only limited applications of these kinds of heterogeneous strategies. The technique we are going to present is studied by Dockener et al. (2000; Example 4.1) where there are two asymmetric players with different objective functions though sharing the same state equation.

The paper is organized as follows: in Section 2, we introduce the concept and formulation of heterogeneous strategy in a general setting. Then, Section 3 presents some situations where anticipating open-loop and non-degenerate Markovian strategies should be played at the same time. Some concluding remarks are given in Section 4.

\section{HETEROGENEOUS STRATEGIES}

Consider a two ${ }^{4}$ players' differential game and assume all functions are continuously differentiable. Each player $i(=1,2)$ chooses $u_{i} \in U_{i}$ (where $U_{i} \in \mathbb{R}$ is the choice space for player $i$ ) to maximize her objective function $\Pi_{i}$ :

$$
\begin{aligned}
& \max _{u_{i}} \Pi_{i}\left(u_{i}, u_{j}\right)=\max _{u_{i}} \int_{0}^{\infty} e^{-r t} f_{i}\left(t, u_{i}, u_{j}, x(t)\right) d t, \\
& i, j=1,2, i \neq j,
\end{aligned}
$$

where player $j^{\prime}$ s strategy $u_{j} \in U_{j}$ is taken as given by player $i$. We assume $x(t) \in X$ is the shared common state of the system ${ }^{5}$ (with $X \in \mathbb{R}$, the state space) and $r$ is a positive constant denoting discounting factor and is the same for both players for simplicity. The state of the system is given by the following differential equation

$$
\dot{x}(t)=g\left(t, u_{1}(t), u_{2}(t), x(t)\right), \forall t \geq 0
$$

\footnotetext{
${ }^{3}$ See Long (2010) for a recent and complete survey

${ }^{4}$ For more than two players' game, given all players share the same information, there would be two groups of players.

${ }^{5}$ Except some players commit to all the state variables, the concept and method may not be analogous if there are different states for different players or multiple states, see for example Reynolds (1987).
} 
with initial condition $x(0)$ given. For simplicity, we also assume both objective functions $f_{i}(\cdot)$ and state function $g(\cdot)$ are at least twice continuously differential.

We impose the heterogeneous strategy in the following sense: player 1 adopts a Markovian strategy and its optimal choice $u_{1}$ depends not only on time $t$, but also on the current state $x$ of the system; while player 2 's optimal strategy $u_{2}$ depends only on time, i.e., there is an irrevocable commitment, which is given based on some anticipating of the rival player's potential Markovian choice. The precise definition of this kind of heterogeneous strategy is the following.

Definition 1 (Heterogeneous Strategic Nash Equilibrium) A 2-tuple $\left(\Psi_{1}, \Psi_{2}\right)$ of functions $\left.\Psi_{1}: X \times 0,+\infty\right) \rightarrow \mathbb{R} \quad$ and $\quad \Psi_{2}:[0,+\infty) \rightarrow \mathbb{R}$, with $\left.\Psi_{1}=\Psi_{1}(x, t), \forall(x, t) \in X \times 0,+\infty\right)$ and $\left.\Psi_{2}=\Psi(t), \forall t \in 0,+\infty\right)$, forms a Heterogeneous Strategic Markovian Nash Equilibrium if, for each player $i$, an optimal control path $u_{i}$ of player $i$ exists and is given by: Markovian strategy for player 1, $u_{1}(t)=\Psi_{1}(x(t), t)$ by knowing player 2's open-loop strategy, and anticipating open-loop strategy for player 2, $\quad u_{2}(t)=\Psi_{2}(t)$ by taking into account player 1's Markovian strategy.

To find the heterogeneous strategy, we depend on some special principle of conjecturing based on Pontryagin's Maximum principle. It is common knowledge that if sufficient concavity conditions are imposed, Pontryagin's Maximum principle can provide not only necessary but also sufficient conditions for each individual player's optimal choice. Thus, if both players adopt strategies based on Pontryagin's Maximum principle, we can write down player i's Hamiltonian function as

$$
H_{i}\left(x, \lambda_{i}, u_{i}, t\right)=f_{i}\left(t, u_{i}, u_{j}^{*}, x\right)+\lambda_{i}(t) g\left(t, u_{i}, u_{j}^{*}, x\right), i=1,2, i \neq j .
$$

Here $u_{j}^{*}$ is player j's optimal choice and taken as given by player $i, \lambda_{i}$ is player i's costate variable.

Theoretically, the usual first order conditions should present the optimal solution, which we denote as: $\Psi_{1}(t)=\Psi_{1}(x(t), t) \quad$ and $\quad \Psi_{2}(t)=\Psi_{2}(x(t), t), \quad \forall t \geq 0$. However, for the case of both players adopt feedback strategies, we face the problem which stated by Kamien and Schwartz (2003, P.275) as: " finding player i's optimal feedback strategy $u_{i}^{*}(x, t)$ requires that player j's optimal feedback strategy $u_{j}^{*}(x, t)$ be known which, in turn, requires that player i's optimal be known, and so on."
Thus, in order to diminish this infinitely looping problem, a special case-heterogeneous strategies can be considered, which are played as following:

Player 1 (the Markovian strategic player) takes player 2's (open-loop) strategy $\Psi_{2}(t)$ as given, and hence, faces the following optimization problem:

$\left\{\begin{array}{l}\max _{u_{1}(x, t)} \int_{0}^{\infty} e^{-r t} f_{1}\left(t, u_{1}(x, t), \Psi_{2}(t), x\right) d t, \\ \text { subjectto } \dot{x}(t)=g\left(t, u_{1}(x(t), t), \Psi_{2}(t), x(t)\right) .\end{array}\right.$

The corresponding current-value Hamiltonian for player 1 is

$$
\begin{aligned}
H_{1}\left(x, \lambda_{1}, u_{1}, t\right)= & f_{1}\left(t, u_{1}(x, t), \Psi_{2}(t), x\right)+\lambda_{1}(t) g\left(t, u_{1}(x, t),\right. \\
& \left.\Psi_{2}(t), x\right),
\end{aligned}
$$

where $\lambda_{1}$ denotes player 1 's costate variable.

Player 2, the open-loop strategy player, applies the conjecturing principle: player 2 guesses that strategy $\Psi_{1}(t)=\Psi_{1}(x(t), t)$ will be replaced by $\Psi_{1}(x, t)$ for any state variable $x \in X$, since player 1 plays Markovian strategy.

Therefore, player 2 (open-loop strategy player), taking player 1's Markovian strategy $\Psi_{1}(x, t)$ as given, faces the following problem:

$$
\left\{\begin{array}{l}
\max _{u_{2}(t)} \int_{0}^{\infty} e^{-r t} f_{2}\left(t, \Psi_{1}(x, t), u_{2}(t), x(t)\right) d t, \\
\text { subjectto } \dot{x}(t)=g\left(t, \Psi_{1}(x(t), t), u_{2}(t), x(t)\right) .
\end{array}\right.
$$

The corresponding current-value Hamiltonian for player 2 is

$$
H_{2}\left(x, \lambda_{2}, u_{2}, t\right)=f_{2}\left(t, \Psi_{1}(x, t), u_{2}, x\right)+\lambda_{2}(t) g\left(t, \Psi_{1}(x, t), u_{2}, x\right),
$$

where $\lambda_{2}$ is the costate variable for player 2 .

Theorem 1 Suppose function $f_{i}(\cdot)$ is strictly concave in control variable $u_{i}$ for $i=1,2$. Denote the solution of optimization problems (3) and (4) as $u_{1}^{*}(x, t)$ and $u_{2}^{*}(t)$, respectively, $\forall x \in X$ and $\forall t \geq 0$. If the two maximized Hamiltonian: for player 1

$H_{1}^{*}\left(x, \lambda_{1}, t\right)=f_{1}\left(t, u_{1}^{*}(x, t), u_{2}^{*}(t), x\right)+\lambda_{1} g\left(t, u_{1}^{*}(x, t), u_{2}^{*}(t), x\right)$

and for player 2

$$
H_{2}^{*}\left(x, \lambda_{2}, t\right)=f_{2}\left(t, u_{1}^{*}(x, t), u_{2}^{*}(t), x\right)+\lambda_{2} g\left(t, u_{1}^{*}(x, t), u_{2}^{*}(t), x\right) .
$$

are both concave in term of state variable $x$, then $\left\{u_{1}^{*}(x, t), u_{2}^{*}(t)\right\}$ forms a pair of non-degenerate 
Markovian Nash Equilibrium for differential game (1) and (2).

Proof. Consider problem (3), the first order condition yields that player 1's choices $u_{1}(x, t)$ is given by the solution of

$$
\partial H_{1} \partial u_{1}=\partial f_{1} \partial u_{1}+\lambda_{1} \partial g \partial u_{1}=0 .
$$

The costate variable $\lambda_{1}$ verifies equation ${ }^{6}$

$$
\dot{\lambda}_{1}(t)=r \lambda_{1}-d H_{1} d x=r \lambda_{1}-\left[\begin{array}{l}
\left(\partial f_{1} \partial x+\lambda_{1} \partial g \partial x\right) \\
+\left(\partial f_{1} \partial u_{1}+\lambda_{1} \partial g \partial u_{1}\right) \partial \Psi_{1}(x, t) \partial x
\end{array}\right],
$$

where $d H_{1} d x$ denotes the total derivative ${ }^{7}$ of $H_{1}$ with respect to $x$ and the last term is equal to zero, by the first order condition (5). Thus, player 1's co-state equation reads

$$
\dot{\lambda}_{1}(t)=r \lambda_{1}(t)-\left(\partial f_{1} \partial x+\lambda_{1}(t) \partial g \partial x\right)
$$

with transversality condition $\lim _{t \rightarrow \infty} e^{-r t} \lambda_{1}(t) x(t)=0$.

Similarly, $u_{2}(t)$, the optimal choices of player 2 , is given by

$\partial H_{2} \partial u_{2}=\partial f_{2} \partial u_{2}+\lambda_{2} \partial g \partial u_{2}=0$.

And, the costate equation is

$\dot{\lambda}_{2}(t)=r \lambda_{2}-d H_{2} d x=r \lambda_{2}-$

$\left[\left(\partial f_{2} \partial x+\partial f_{2} \partial u_{1} \partial \Psi_{1}(x, t) \partial x\right)+\lambda_{2}\left(\partial g \partial x+\partial g \partial u_{1} \partial \Psi_{1}(x, t) \partial x\right)\right]$

or equivalently,

$$
\dot{\lambda}_{2}(t)=r \lambda_{2}-\left[\begin{array}{l}
\left(\partial f_{2} \partial x+\lambda_{2} \partial g \partial x\right)+ \\
\left(\partial f_{2} \partial u_{1}+\lambda_{2} \partial g \partial u_{1}\right) \partial \Psi_{1}(x, t) \partial x
\end{array}\right] .
$$

The associated transversality condition is $\lim _{t \rightarrow \infty} e^{-r t} \lambda_{2}(t) x(t)=0$.

Remark. Equation (8) differs from (6) due to the fact that in (6) the first order condition (5) can be applied; while in (8), that is not possible.

Denote the solution of (5) and (7) as $u_{1}^{*}=\Psi_{1}^{*}(x, t), \forall(x, t) \in X \times[0, \infty)$ and $u_{2}^{*}=\Psi_{2}^{*}(t), \forall t \geq 0$,

${ }^{6} \mathrm{~A}$ similar notation can also be found in Itaya and Shimomura (2001). However, without guessing process, they can not go further than just writing down the functional form of first order conditions. See more systematic statement for the general case in Kamien and Schwartz (2003, Page 275).

'The state variable affects the current-value Hamiltonian via two different ways: the direct impacts by the state equation and indirect impacts due to the strategy of the other player. respectively. To be more precise $\Psi_{1}^{*}(x, t)$ is a function of state $x$, the costate variable evaluated at time $t$; and $\Psi_{2}^{*}(t)$ is a function of state and costate variables both evaluated at $t$, thus, $\Psi_{1}^{*}(x, t)=\Psi_{1}^{*}\left(x, \lambda_{1}(t), t\right)$ and $\Psi_{2}^{*}(t)=\Psi_{2}^{*}\left(x(t), \lambda_{2}(t), t\right)$. Substituting these two into the Hamiltonian, we can readily check that the maximized Hamiltonian $H_{1}^{*}\left(x, \lambda_{1}, t\right)$ and $H_{2}^{*}\left(x, \lambda_{2}, t\right)$ are given by

$$
\begin{array}{r}
H_{1}^{*}\left(x, \lambda_{1}, t\right)=f_{1}\left(t, \Psi_{1}^{*}\left(x, \lambda_{1}, t\right), \Psi_{2}^{*}\left(x, \lambda_{2}, t\right), x\right)+ \\
\lambda_{1} g\left(t, \Psi_{1}^{*}\left(x, \lambda_{1}, t\right), \Psi_{2}^{*}\left(x, \lambda_{2}, t\right), x\right)
\end{array}
$$

and

$$
\begin{aligned}
H_{2}^{*}\left(x, \lambda_{2}, t\right)= & f_{2}\left(t, \Psi_{1}^{*}\left(x, \lambda_{1}, t\right), \Psi_{2}^{*}\left(x, \lambda_{2}, t\right), x\right)+ \\
& \lambda_{2} g\left(t, \Psi_{1}^{*}\left(x, \lambda_{1}, t\right), \Psi_{2}^{*}\left(x, \lambda_{2}, t\right), x\right) .
\end{aligned}
$$

Given the maximized Hamiltonian are both concave with respect to the state variable $x$. Then, by Theorem 3.2 (Dockner et al, 2000), $u_{i}^{*}(t) \quad(i=1,2)$ are optimal paths. Thus, the solution $\left\{u_{1}^{*}(x, t), u_{2}^{*}(t)\right\}, \forall x \in X$ and $\forall t \geq 0$ form a pair of non-degenerate Markovian Nash Equilibrium.

Finally, substituting $u_{1}^{*}=\Psi_{1}^{*}(x, t)$ and $u_{2}^{*}=\Psi_{2}^{*}(t)$ into the canonical system: state equation (2), two costate equations (6) and (8), we can obtain the solution for the whole trajectory path of the differential game. That finishes the proof.

Remark. It is easy to see if the Markovian player's strategy is independent of the state $x$, then the above Heterogeneous Strategic Markovian Nash equilibrium is just degenerate open-loop Nash equilibrium.

Nonetheless, in Heterogeneous Strategic Markovian Nash equilibrium, the open-loop player differs from the classical case where both players adopt open-loop strategies. Under current setting, the open-loop strategic player corrects, though at the beginning of the game by some guessing principle, her strategy based on the possible updating the rival player may take. Thus, the open-loop strategy player is not completely passive, rather in an anticipating defensive position. We call this kind of open-loop strategy as anticipating open-loop strategy ${ }^{8}$. This way of constructing strategy gives possibility of subgame perfection. ${ }^{9}$

${ }^{8}$ Our definition of anticipating strategy is different from the non-anticipating strategy defined in Chapter 8, Dockner et al. (2000), in which the nonanticipating strategy means that a feasible strategy for an optimal action at time $t$ can only depend on information that has been revealed by that time, rather than the realization of future time. Our definition of anticipating focus on the anticipating of the rival's strategic form (that is, knowing the rival player will update her strategy and play Markovian strategy), rather than on the future information.

${ }^{9}$ Of course, open-loop strategy may be subgame perfect, see Reynold (1987). 
We close this section with a brief remark concerning the subgame perfection of stationary Markovian Nash Equilibrium in an autonomous system.

Theorem 2 Suppose the differential game (3) and (4) defined on time interval $[0, \infty)$ and suppose the system under study is autonomous. Then under the assumption of the Theorem 1, the pair of stationary strategy, $\left(\bar{u}_{1}^{*}=\Psi_{1}^{*}(\bar{x}), \bar{u}_{2}^{*}=\Psi_{2}^{*}(\bar{x})\right)$ is subgame perfect heterogeneous strategic Markovian Nash equilibrium as long as it does not depend on the initial condition $x(0)$.

Proof. The reason for subgame perfection follows the remark of Dockner et al (2000, P.105)- if an autonomous differential game is defined on the time interval $[0, \infty$ ), then its subgame is equivalent (in fact, identical) to the original game. It follows from the definition of subgame perfect Nash equilibrium that any stationary Markovian Nash Equilibrium is subgame perfect, provided it is independent of the initial state $x(0)$.

Obviously, if the system is non-autonomous, this results may not be true, even the stationary strategy is independent of the initial state.

Remark. The subgame perfection is most probably only true in the set of heterogeneous strategic Markovian Nash equilibrium, which is a subset of the set of infinitely many non-degenerate Markovian Nash equilibrium.

The economic interpretation is the following: Player 2 adopts an anticipating open-loop strategy which is based on guessing the Markovian strategy of player 1 . If player 1 plays subgame perfect stationary strategy, player 2, as a by-product, also plays subgame perfect strategy. The reason is that the guessing process includes all the information about what player 1's optimal strategy will be. This differs from the case where both players adopt open loop strategies. Thus, the updating information is embodied in the guessing process rather than losing in commitments.

\section{EXAMPLES AND APPLICATIONS}

In this section, we provide some examples where asymmetric situations appear and heterogeneous strategy spaces should be adopted by the players. The order of examples are the following: Example 1 and 2 present the situation where the two players enjoy the same information set, however, one does not have the same capacity of taking the same strategy as her rival.
Example 1 comes directly from the recent publication of Han et al. (2014) where they also study the difference between heterogeneous strategies and homogenous ones. Example 2 is a modification of Dawid and Feichtinger (1996). Some more potential applications in this case are also presented after Example 2. Example 3 and 4 are the case where the two players are symmetric or identical in obtaining information of the state of the world and having the same capacity of making decision, however, due to social choices, political considerations or other constraints, one player commits to and keeps on a strategy, while the other player updates her strategy based on the state of the world. This is the case we called unilateral commitment in differential game.

This list of real world situations in which dynamic heterogeneous strategies are played is not exhaustive. Further applications and potential examples will be mentioned again in the conclusion.

\subsection{Same Information Set but Different Strategy Spaces}

\section{Example 1. Dynamic Tax Competition between Unequal Size Jurisdictions-Han et al. (2014)}

Most models in the tax competition literature are static. Though Zissimos and Wooders (2008) have called for the need of dynamic studies of tax competition, Han et al. (2014) is one of the very few exceptions studying tax competition under a dynamicstrategic setting. They assume that a small economy and a large one enter a dynamic tax-and-infrastructurecompetition game where the size asymmetry of these two economies play an essential role.

As they argued, small-in-size is a natural disadvantage for most of the small economies, but sometimes small-in-size can be considered an as asset (Kuznets, 1960; Easterly and Kray, 2000) given the economic success of many micro-states. Han et al (2014) argue that small states are more flexible in their political decision making than much larger countries (Streeten, 1993); for example, problems related to collective action can be solved more easily in small countries. These attributes facilitate greater singlemindedness and focus on economic policy-making and promote a more rapid and effective response to exogenous change (Armstrong and Read, 1995).

Han et al. (2014) introduce dynamic firm relocation process via location attachment assumption. More precisely, they assume that the one person one capital 
firm produces net $q+a_{i}$ units final goods and sold in world competitive market with price normalized to 1 . Here, $q$ presents private firm's productivity and $a_{i}$, $i=1,2$, reads country $i$ 's specific productivity enhancing public goods.

Consider an entrepreneur in the small country, if she invests at home, the profit is

$\pi_{1}(t)=q(t)+a_{1}(t)-T_{1}(t)$

and if she invests abroad, profit is then

$\pi_{2}(t)=q(t)+a_{2}(t)-T_{2}(t)-k \cdot x(t)$

with $T_{i}(t)$ tax rate in country $i=1,2$. Here, mobility cost is $k \cdot x(t)$ with unit mobility cost $k$ and distance to frontier $x$.

Similar arguments are also true for firms originally located in big country. Thus, we can obtain the indifferent firm is located at

$x\left(t, a_{1}, a_{2}, T_{1}, T_{2}\right)=\frac{a_{2}(t)-T_{2}(t)}{k}-\frac{a_{1}(t)-T_{1}(t)}{k}$.

If denote number of firms at time $t$ located in the small economy as $S_{1}(t)=S(t)$ and the number of firms in the large economy is then $S_{2}(t)=1-S(t)$ by assuming that total number of firms is constant and normalized to 1 . Thus, law of motion of number of firm in the small economy is governed by

$\dot{S}(t)=-x=\frac{a_{1}(t)-T_{1}(t)}{k}-\frac{a_{2}(t)-T_{2}(t)}{k}$.

Furthermore, both small and large economies' policy makers choose tax $T_{i}(t)$ and public goods $a_{i}(t)$ simultaneously to maximize tax revenue:

$J_{i}=\max _{a_{i}, T_{i}} \int_{0}^{+\infty} e^{-r t}\left(\sqrt{S_{i} T_{i}}-\frac{\beta_{i}}{2} a_{i}^{2}\right) d t, i=1,2$,

subject to dynamic relocation of firms (9).

Though, mathematically, the optimization problem for both small and big economies are the same, they may react differently due to their size differentiation. A given number of firms may be just a small sector in the big economy, but could be the whole or the main industry of a small economy. Thus, the degree of competition and the importance of competition differ between the big and small economies. This dissimilarity induces the two economies behave accordingly. In order to survive, the small economy must react collectively and more rapidly depending on the changes of situations - Markovian strategy; while the large economy can afford to follow plans though taking into account the possible strategic actions the small economy may take - anticipating open-loop strategy.

Han et al. (2014) find that the extra flexibility in policy making- taking Markovian strategy - is very essential for the surviving of small states while competing with big folks.

\section{Example 2. Optimal Allocation of Drug Control Efforts}

Dawid and Feichtinger (1996) provide a dynamic drug control problem with two players, i.e., drug dealers and the government. Thus, the players are asymmetric.

The model is the following: both government and drug dealers maximize their respective objectives. The drug dealers choose, $u>0$, the effort of the dealers, which they interpret as the time the dealer spends in the street in order to attract new customers; and government chooses, $v>0$, the whole expense spent to deal with drug problem. Denote $x(t) \in[0, \bar{x}]$ as drug user at time $t$, with given fixed upper bound $\bar{x}>0$.

Dawid and Feichtinger (1996) assume that the growth of the stock of drug users is governed by three forces: activities of the drug dealers, death and treatment of drug users. Denote $g(x)$ as a growth function with the characteristic notions of a diffusion dynamics of drug user, $d$ as death rate and hence, the motion of drug user follows:

$\left\{\begin{array}{l}\dot{x}=g(x) \sqrt{u}-d x-f(x) \sqrt{\phi v}, \\ x(0)=x_{0} \in[0, \bar{x}], \phi \in[0,1], d>0 .\end{array}\right.$

Here, function $f(x) \sqrt{\phi v}$ measures the treatment effects, with $\phi \in[0,1]$ fraction of budget for drug control invested in treatment, $(1-\phi) v$ the fraction of budget of crackdown on dealers, and $f \in C^{1}[0, \bar{x}]$ checks: $f(0)=0, f^{\prime}(x)>0$ and $(f(x) / x)^{\prime}<0$.

The objective of drug dealer is

$\max _{u \in[0, \infty)} J_{d}=\int_{0}^{\infty} e^{-r_{d} t}\left[U(x)-C_{d}(u,(1-\phi) v)\right] d t$,

subject to constraint (10), where function $U(\cdot)$ is dealer's income and function $C_{d}(\cdot)$ describes the damage for the whole class of dealers caused by government law enforcement actions. 
The problem of government is the cost produced by the drug users, including the direct cost, $D(x)$, and the effort of controlling the drug problem, $C_{g}(v)$. Thus, the objective of government is:

$\max _{v \in[0, \infty)} J_{g}=\int_{0}^{\infty} e^{-r_{g} t}\left[-D(x)-C_{g}(v)\right] d t$,

subject to constraint (10).

Dawid and Feichtinger (1996) develop an explicit solution of the stationary feedback strategies where both players play Markovian strategies. Arguably, though both government and drug dealers have the same information as to the number of drug users, they have different objective and different constraint on taking their strategic actions. Thus, different strategic spaces may be more proper: government's treatment and law enforcement are based on law and/or regulations while the drug dealers act in ways to avoid government control. Given the law and regulations are transparent and announced by the government( if we do not consider policemen's action) while drug dealers do not communicate on their actions and strategies, heterogeneous strategies could be applied in such a context: the drug dealers change their strategies and efforts based on the reality-Markovian strategy, while government action is more open-loop by taking into account the drug dealers' effort, thus anticipating openloop strategy. Therefore, the two players reach to one heterogeneous strategic Nash Equilibrium.

We close this subsection by noticing that these kinds of examples could also come from the dynamic competition of oligopoly with competitive fringes. In a static setting, Shimomura and Thisse (2012) study asymmetric competition among big and small firms, in which the few big commercial or manufacturing firms are able to affect the market outcome, and a myriad of small family-run businesses with very few employees has a negligible impact on the market. In a generalequilibrium setting, they demonstrate that (abstract on Page 1) "due to the higher toughness of the market, the entry of big firms leads them to sell more through a market expansion effect, which is generated by the exit of small firms." Thus, asymmetric strategies are played at the same time depending on the market power. It would be interesting to study the dynamics of this kind of games and its long-run outcome under a differential game where the market share (such as in Han et al. 2014) or the goods' prices (such as in Fershtman and Kamien, 1987, 1990) could serve as the state variable. Benchekroun et al. (2009) indeed work out a similar asymmetric situation "on nonrenewable resource oligopoly". Nonetheless, they only study the outcome where both players using open-loop strategies, thus the asymmetric marketing power of strategic choice can not be shown explicitly.

\subsection{Symmetric Players but Heterogeneous Strategies}

\section{Example 3. Transboundary Pollution- Unilateral Commitments}

This example relates to the international $\mathrm{CO}_{2}$ emission control problem. Since the seminal paper of Dockner and Sorger (1996), there have been various contributions using differential games to study transboundary pollution control problems. However, most of them have simply ignored open-loop strategies. The main reason is that it was thought that players are too naive to play open-loop strategies as they do not use any information acquired during the game and, consequently, do not respond to changes in the current pollution stock. In the context of climate change and policies that have been implemented to its mitigation, it is important to distinguish between countries which have taken binding commitments to stabilize/reduce their Greenhouse Gas (GHG) emissions, such as in the Kyoto Protocol or the EU 2007 Energy Package, and countries which have proposed more flexible approaches, based on regular updates of the targets to reach, according to current states of the world.

For this kind of problem, the EU, under the Kyoto Protocol or its own unilateral policies, makes commitments about emission reductions from which they cannot deviate, while the USA and China, on the contrary, can deviate from their commitments (or do not have any commitment at all) and regularly revise their targets and policies. In the first case, we refer to openloop strategies and in the second case to Markovian strategies. And, obviously, the classical method, where either both players adopt open-loop strategies or both play Markovian strategies, is not the proper one in these sorts of circumstances.

Bertinelli et al. (2015) present one of this type of example. The choices of different players could be the abatement efforts or $\mathrm{CO}_{2}$ reduction and the common state will be the environmental quality or $\mathrm{CO}_{2}$ stock. More precisely, the possible model is the following.

These two players undergo the same pollution state, $x(t)$, which is given by the following equation

$\dot{x}(t)=E(t)-\left(u_{i}+u_{j}\right) \sqrt{x(t)}-\delta x(t), t \geq 0$, 
where the initial condition $x(0)$ is a given positive constant, and parameter $\delta \in[0,1]$ measures the pollution absorption rate of nature. $E(t)=E_{i}(t)+E_{j}(t)$ is a known positive function of pollution emissions.

The two countries' policy makers need to choose their abatement rate $u_{l}, l=i, j$, to maximize their utility

$$
\max _{u_{l}} \int_{0}^{\bar{T}} e^{-r_{l} t}\left(-x(t)-\alpha_{l} 2 u_{l}^{2}\right) d t+S(x(\bar{T})), l=i, j,
$$

subject to the state constraint (13), where $r_{l} \in[0,1)$ is the time preference parameter, $\alpha_{l}$ is a positive, constant adjustment cost coefficient. We consider $\bar{T} \leq \infty$, where $S(x(\infty))=0$ and in finite time $\bar{T}=T$, $S(x(T))$ is a given known positive function, with $S_{x}<0$.

Furthermore, $x(\bar{T})$ is the final target of the pollution state of the world, where both players agree on a final date $T$.

Most probably, as long as $T<\infty$, the heterogeneous strategy is not subgame perfect, which coincides with the tragedy-of-the-commons' outcome, this unilateral decision should have worsened the welfare of EU citizens.

\section{Example 4. Unilateral Production Restrictions in a Dynamic Duopoly}

Benchekroun (2003) presents a model where several countries share access to a productive asset and the agreements upon the exploitation of the asset are very hard to reach.

In more detail, he assumes that firm $i(=1,2)$ maximizes its own discounted sum of instantaneous profits:

$J^{i}=\int_{0}^{\infty} P\left(q_{1}(t)+q_{2}(t)\right) q_{i}(t) e^{-r t} d t$,

subject to the law of motion of asset stock

$\dot{S}=F(S)-q_{1}(t)-q_{2}(t), S(0)=S_{0}$ given.

Here, parameter $r$ is time preference, $q_{i}$ is quantity of output of firm $i$ from country $i$, price $P(q)$ is inverse demand function, $S$ reads stock of productive asset and function $F(S)$ presents the production of stock, such as fish population.

Benchekroun (2003) first presents the results of laissez-faire closed-loop Nash equilibrium, then he goes one step further consider a situation where one player wishes to increase the asset's stock in the long run. Given "no agreement between the two countries is possible: country one can only regulate its own firm's production" (Page 222), thus " country one implements unilaterally a production restriction". In doing so, Benchekroun (2003) first "supposes that country one constrains its firm to adopt" an exogenously given strategy based on the above calculated laissez-faire closed-loop Nash equilibrium strategy; then the firm two's best response, to the given firm one's strategy, is calculated. Thus, long-run steady states studies, under the two different strategy sets, are possible and comparison outcomes are presented.

However, the given constrained strategy of country one is more based on common sense without justification, which may not be optimal choice of the country one. Thus, most probably, the pair of strategies is not one Nash equilibrium. It is plausible and interesting to study this kind of setting but apply heterogeneous strategic Nash equilibrium and compared with laissez-faire closed-loop Nash equilibrium.

\section{CONCLUDING REMARKS}

Some particular heterogeneous strategies are introduced in various kinds of differential games. The heterogeneity means that one player adopts an anticipating open-loop strategy while the other player takes on a non-degenerate Markovian strategy - thus the strategy spaces are heterogeneous. The key idea is the guessing of the rival's strategies and the construction of strategy could be specially useful to the study of asymmetric players' differential games.

The novelty of this kind of strategy is twofold. On the one hand, it offers another - except the standard Markovian strategy for all players - stationary subgame perfect non-degenerate Markovian Nash equilibrium for an autonomous system with infinite-horizon. On the other hand, via Hamiltonian, it can characterize the whole trajectory, which is especially useful in the case of asymmetric players' non-linear-quadratic differential games. However, it may be hard to prove its subgame perfection. The short-run trajectory may be very different from the long-run stationary solution. Nevertheless, the strategy's finite-horizon disadvantage itself offers useful information to some differential games where unilateral commitments happen. For instance, in the case of environmental problem we mentioned in Section 3, a short-run committed policy is most likely not subgame perfect and hence, introducing this kind of policy will not only increase the free riding problem, but also hurt the player's own welfare. 
For future research, some special care should be given to the case where multiple states arise, such as Reynolds (1987). It may be the case that even committed players only commit to some state variables rather than to all. In case of multiple instruments' competition, some of the control variables depend on some states and time while the other control variables depend on time only. And these differ from our setting here.

\section{ACKNOWLEDGEMENT}

I gratefully acknowledge the useful and inspiring discussions with Luisito Bertinelli, Raouf Boucekkine, Herbert Dawid, Engelbert Dockner, Gustav Feichtinger, Patrice Pieretti, Jennifer Reinganum, loana Salagean, and Gerhard Sorger; as well as the comments from 13th Viennese Workshop on Optimal Control and Dynamic Games, 2015.

\section{REFERENCES}

Armstrong, H.W. and R. Read. 1995. Western European Micro-states and EU autonomous Regions: the Advantages of Size and Sovereignty. World Development, 23(7), 1229-1245. http://dx.doi.org/10.1016/0305-750X(95)00040-J

Basar, T. 1976. On the uniqueness of the Nash solution in linearquadratic differential games. International Journal of Game Theory, 5(2/3), 65-90. http://dx.doi.org/10.1007/BF01753310

Benchekroun, H. 2003. Unilateral production restrictions in a dynamic duopoly. Journal of Economic Theory, 111, 214-239. http://dx.doi.org/10.1016/S0022-0531(03)00090-5

Benchekroun, H., A. Halsema and C. Withagen. 2009. On nonrenewable resource oligopolies: the asymmetric case. Journal of Economic Dynamic and Control, 33, 1867-1879. http://dx.doi.org/10.1016/j.jedc.2009.03.008

Bertinelli, L., L. Marchiori, A. Tabakovic and B. Zou. 2015. Transboundary Pollution Abatement: The Impact of Unilateral Commitment in Differential Games. CREA DP 2015-02.

Dawid, H. and G. Feichtinger. 1996. Optimal allocation of drug control efforts: A differential game anaylsis. Journal of Optimization Theory and Application, 91(2), 279-297. http://dx.doi.org/10.1007/BF02190097

Dockner, E. and G. Sorger. 1996. Existence and properties of equilibria for a dynamic game on productive assets. Journal of Economic Theory, 71, 209-227.

http://dx.doi.org/10.1006/jeth.1996.0115

Dockner, E., Jorgensen S., Van Long N., and G. Sorger. 2000. Differential games in economics and management: Cambridge University Press.

http://dx.doi.org/10.1017/CBO9780511805127
Easterly, W. and A. Kray. 2000. Small states, small problems ? Income, growth, and volatility in small states. World Development, 28 (11): 2013-2027. http://dx.doi.org/10.1016/S0305-750X(00)00068-1

Fershtman, Ch. and M. Kamien. 1987. Dynamic duopolistic competition with sticky prices. Econometrica, 55(5), 11511164.

http://dx.doi.org/10.2307/1911265

Fershtman, Ch. and M. Kamien. 1990. Turnpike Properties in a Finite-Horizon Differential Game: Dynamic Duopoly with Sticky Prices. International Economics Review, 31(1), 49-60. http://dx.doi.org/10.2307/2526627

Han, Y., Pieretti P., S. Zanaj and B. Zou. 2014. Asymmetric competition among Nation States-A differential game approach. Journal of Public Economics, 119, 71-79. http://dx.doi.org/10.1016/j.jpubeco.2014.07.008

Itaya, J. and K. Shimomura. 2001. A dynamic conjectural variations model in the private provision of public goods: a differentia game approach. Journal of Public Economics, 81, 153-172. http://dx.doi.org/10.1016/S0047-2727(00)00111-0

Kamien, M. and N. Schwartz. 2003. Dynamic optimization. 2nd Edition and 7th Impression: Elsevier, North Holland.

Kuznets, S. 1960. Economic Growth of Small Nations. In The Economic Consequences of the Size of Nations. ed. E.A.G. Robinson. Proceedings of a Conference Held by the International Economic Associations: MacMillan, Toronto. http://dx.doi.org/10.1007/978-1-349-15210-0 2

Long, N. 2010. A surveyy of Dynamic Games in Economics: World Scientific. http://dx.doi.org/10.1142/9789814293044

Reinganum, J. and N. Stokey. 1985. Oligopoly extraction of a common perperty natureal esource: The importantce of the period of commitment in dynamic games. International Economic Review,39(1), 161-173. http://dx.doi.org/10.2307/2526532

Reinganum, J. 1981. On the diffusion of new technology: A game theoretic approach. The Review of Economic Studies, 48(3), 395-405. http://dx.doi.org/10.2307/2297153

Reynolds, S. 1987. Capacity investment, preemption and commitmnet in an infinite horizon model. International Economic Review, 28(1), 69-88. http://dx.doi.org/10.2307/2526860

Shimomura, K. and J. F. Thisse. 2012. Competition among the big and the small. RAND Journal of Economics, RAND Corporation, 43(2), 329-347.

Sorger, G. 2015. Dynamic Economic Analysis: Deterministic Models in Discrete Time: Cambridge University Press.

Streeten, P. 1993. The Special Problems of Small Countries. World Development, 21(2): 197-202. http://dx.doi.org/10.1016/0305-750X(93)90014-Z

Zissimos, B. and M. Wooders. 2008. Public good differentiation and the intensity of tax competition. Journal of Public Economics, 92, 1105-1121. http://dx.doi.org/10.1016/j.jpubeco.2007.09.009

\section{DOI: http://dx.doi.org/10.6000/1929-7092.2016.05.15}

(c) 2016 Benteng Zou; Licensee Lifescience Global.

This is an open access article licensed under the terms of the Creative Commons Attribution Non-Commercial License (http://creativecommons.org/licenses/by-nc/3.0/) which permits unrestricted, non-commercial use, distribution and reproduction in any medium, provided the work is properly cited. 